\begin{tabular}{|c|c|c|}
\hline Beitr. Ent. & Keltern & ISSN 0005-805X \\
\hline $\mathbf{6 2}(2012) 1$ & S. $77-102$ & 15.05 .2012 \\
\hline
\end{tabular}

\title{
Biodiversità delle Aleocharinae della Cina: Lomechusini e Thamiaraeini ${ }^{1}$
}

\section{(Coleoptera, Staphylinidae)}

Con 69 figure

\section{Roberto Pace}

\section{Summary}

Description of 21 new species of the tribes Lomechusini and Thamiaraeini belonging to the genera Orphnebius (O. uncinatus n. sp.), Tetrabothrus (T. chinensis n. sp.), Drusilla (D. damingensis n. sp., D. cenwanglaomontis n. sp., D. gilva n. sp., D. sinicola n. sp.), Zyras (Z. qingchengensis n. sp., Z. sichuanorum n. sp., Z. yongshengensis n. sp., Z. diaoluomontis n. sp., Z. fellowesi n. sp.), Pedinopleurus (P. jiuwanmontis n. sp.), Eutaenoglossa (E. schuelkei n. sp.), Platorischna (P. sinensis n. sp., P. sichuanensis n. sp.), Lasiokoryphina n. gen. (L. sinica n. sp.), Medeterusa (M. chinensis n. sp.), Mimacrotona (M. chinensis n. sp.), Mimoxypoda (M. sinica n. sp., M. sinorum n. sp.), Litoglossa (L. chinensis n. sp.). Each new species is illustrated and compared with similar species. Additional distributional data are reported for 14 species. The new genus Lasiokoryphina is similar to Platorischna. Drusilla hanoiensis PAśnIK, 2000 is placed in synonymy with Zyras rufithorax Cameron, 1930. New combination is proposed for 1 species.

\section{Key words}

Coleoptera, Staphylinidae, Aleocharinae, taxonomy, China

\section{Zusammenfassung}

21 neue Arten der Tribus Lomechusini und Thamiaraeini aus den folgenden Gattungen werden beschrieben: Orphnebius (O. uncinatus n. sp.), Tetrabothrus (T. chinensis n. sp.), Drusilla (D. damingensis n. sp., $D$. cenwanglaomontis $\mathrm{n}$. sp., D. gilva n. sp., D. sinicola n. sp.), Zyras (Z. qingchengensis $\mathrm{n}$. sp., Z. sichuanorum n. sp., Z. yongshengensis n. sp., $Z$. diaoluomontis n. sp., Z. fellowesi n. sp.), Pedinopleurus (P. jiuwanmontis n. sp.), Eutaenoglossa (E. schuelkei n. sp.), Platorischna (P. sinensis n. sp., P. sichuanensis n. sp.), Lasiokoryphina n. gen. (L. sinica n. sp.), Medeterusa (M. chinensis n. sp.), Mimacrotona (M. chinensis n. sp.), Mimoxypoda (M. sinica n. sp., M. sinorum n. sp.), Litoglossa (L. chinensis n. sp.). Jede neue Art wird illustriert und mit ähnlichen Arten verglichen. Zusätzlich werden Daten zur Verbreitung von 14 Arten gebracht. Die neue Gattung Lasiokoryphina ist ähnlich Platorischna. Drusilla hanoiensis PaśNIK, 2000 wird in die Synonymie von Zyras rufithorax CAMERoN, 1930 gestellt. Eine Art wird neu kombiniert.

\section{Riassunto}

Descrizione di 21 nuove specie delle tribù Lomechusini e Thamiaraeini appartenenti ai generi Orphnebius (O. uncinatus n. sp.), Tetrabothrus (T. chinensis n. sp.), Drusilla (D. damingensis n. sp., D. cenwanglaomontis n. sp., D. gilva n. sp., D. sinicola n. sp.), Zyras (Z. qingchengensis n. sp., Z. sichuanorum n. sp., Z. yongshengensis n. sp., Z. diaoluomontis n. sp., Z. fellowesi n. sp.), Pedinopleurus (P. jiuwanmontis n. sp.), Eutaenoglossa

${ }^{1} 249^{\circ}$ Contributo alla conoscenza delle Aleocharinae. 
(E. schuelkei n. sp.), Platorischna (P. sinensis n. sp., P. sichuanensis n. sp.), Lasiokoryphina n. gen. (L. sinica n. sp.), Medeterusa (M. chinensis n. sp.), Mimacrotona (M. chinensis n. sp.), Mimoxypoda (M. sinica n. sp., M. sinorum n. sp.), Litoglossa (L. chinensis n. sp.). Ogni nuova specie è illustrata e comparata con specie simili. Sono riferiti dati aggiuntivi sulla distribuzione per 14 specie. Il nuovo genere Lasiokoryphina è affine a Platorischna. Drusilla hanoiensis Paśnik, 2000 è posta in sinonimia di Zyras rufithorax Cameron, 1930. E' data una nuova combinazione.

\section{Introduzione}

In questi ultimi anni le ricerche entomologiche si sono particolarmente intensificate in Cina, con conseguente incremento di pubblicazioni riguardanti lo studio tassonomico di Aleocharinae. In questo ambito si inserisce anche il presente lavoro che è un contributo a una sempre migliore conoscenza sulla tassonomia di questa sottofamiglia degli Staphylinidae della Cina.

\section{Materiale e metodo}

Gli esemplari del presente lavoro sono stati raccolti recentemente (1997, 1999, 2001, 2003) dai colleghi studiosi di Staphylinidae Dr. Aleš Smetana di Ottawa, Guillaume de Rougemont di London e Michael Schülke di Berlino. Comprende anche le raccolte di Andreas Pütz a me affidate in studio dal Dr. Olaf Jäger dello Senckenberg Museum für Tierkunde di Dresda.

Anche lo studio tassonomico delle specie cinesi della sottofamiglia Aleocharinae, come quello d'altre regioni zoogeografiche, presenta seri problemi interpretativi che sono risolti in gran parte grazie all'esame della forma dell'organo copulatore maschile (edeago) e della spermateca.

Gli esemplari sono stati dissezionati con lo scopo di includere le strutture genitali in balsamo del Canadà (su piccoli rettangoli trasparenti di materiale di plastica, che accompagnano gli esemplari). Le strutture genitali sono state studiate con l'uso di un microscopio composto fino a 450 ingrandimenti e disegnate per mezzo di oculare a reticolo. Le misurazioni sono state eseguite mediante l'ausilio di un oculare con scala micrometrica. I caratteri dell'habitus sono stati osservati con microscopio stereoscopico fino a 100x. Tutti i disegni delle tavole e le foto sono stati da me eseguiti fino alla fase finale. Le tavole sono state da me composte al computer.

\section{Acronimi}

Gli holotypi e paratypi delle nuove specie sono conservati nei seguenti istituti:

SDEI Senckenberg Deutsches Entomologisches Institut di Müncheberg

SMTD Senckenberg Museum für Tierkunde, Dresden

MNHU Museum für Naturkunde Humboldt Universität, Berlin

IRSNB Institut royal des Sciences naturelles de Belgique, Bruxelles

MNHL Museo di Storia naturale di Londra

MHNG Museo di Storia naturale di Ginevra

MRSNT Museo Regionale di Scienze Naturali di Torino

Altri paratypi in collezione Smetana. 
Lomechusini Fleming, 1821

Orphnebius Motschulsky, 1858

\section{Orphnebius hauseri EPPELSHEIM, 1895}

Orphnebius hauseri EPPELsheim: 54

$10^{\star}$, China: Sichuan, Gongga Shan, Moxi, 1300 m, 11.VII.1996, leg. A. Smetana; 1 , China: N-Yunnan, Zhongdian Co., $46 \mathrm{~km}$ SSE Zhongdian, $27^{\circ} 27.0^{\prime} \mathrm{N} 99^{\circ} 54.7 \mathrm{E}, 3050-3100 \mathrm{~m}$, 17.VIII.2003, leg. A. Smetana.

\section{Distribuzione:}

Specie finora nota dell'India e Nepal.

\section{Orphnebius uncinatus n. sp.}

(Figg. 17-19)

\section{Materiale tipico:}

Holotypus ơ, China: Sichuan, Gongga Shan, Moxi, 1300 m, 11.VII.1996, leg. A. Smetana (MHNG).

\section{Descrizione:}

Lunghezza 3,4 mm. Corpo lucidissimo. Avancorpo nero-bruno, addome giallo-rossiccio, antenne brune con i tre antennomeri basali giallo-rossicci, zampe giallo-rossicce. Secondo antennomero più corto del primo, terzo più lungo del secondo, quarto lungo quanto largo, quinto a decimo trasversi. Occhi più lunghi delle tempie. Reticolazione del corpo assente. Capo con solo quattro punti superficiali presso gli occhi, due per parte. Qualche punto poco evidente sul pronoto. Punteggiatura delle elitre fine e rada. Uroterghi liberi concavi e quasi nudi, quinto urotergo libero del ơ solcato da strie lingitudinali superficiali. Edeago figg. 18-19.

\section{Comparazioni:}

Per la forma dell'edeago e delle antenne la nuova specie è comparabile con $O$. incisus PACE, 2000 della Thailandia. Se ne distingue per l'apice dell'edeago, in visione laterale, ricurvo (rettilineo in incisus) e per la debole incisione mediana apicale, in visione ventrale, (profonda incisione in incisus). Il quinto urotergo libero del $\sigma^{\top}$ è striato nella nuova specie, punteggiato in incisus.

\section{Etimologia:}

La nuova specie prende nome dalla forma dell'apice dell'edeago, in visione laterale.

\section{Tetrabothrus Bernhauer, 1915}

\section{Tetrabothrus chinensis n. sp.}

(Figg. 1 e 20-21)

\section{Materiale tipico:}

Holotypus ơ, China: border Shaanxi, Daba Shan pass, $20 \mathrm{~km}$ SSE Zhenping, 1700-1800 m, $31^{\circ} 44^{\prime} \mathrm{N} 109^{\circ} 35^{\prime} \mathrm{E}, 12$.VII.2001, leg. A. Smetana (SDEI). 


\section{Descrizione:}

Lunghezza 5,5 mm. Corpo lucido e nero-bruno, addome giallo-rossiccio, antenne brune, zampe brune con base dei femori gialla. Secondo antennomero più corto del primo, terzo più lungo del secondo, quarto a decimo fortemente trasversi. Occhi lunghi quanto le tempie. Reticolazione del corpo assente. Punteggiatura del capo fine, quella del pronoto superficiale, quella delle elitre quasi invisibile. Edeago figg. 20-21.

\section{Comparazioni:}

Per la forma dell'edeago la nuova specie è simile a T. validus Maruyama \& Kishimoto, 1999 del Giappone. Se ne distingue per il profilo ventrale dell'edeago sinuato (arcuato in validus) e per l'armatura genitale interna dell'edeago lunga e stretta (assente in validus).

\section{Etimologia:}

La nuova specie ovviamente prende nome dalla Cina.

\section{Amaurodera Fauvel, 1905}

\section{Amaurodera smetanai PACE, 1992}

Amaurodera smetanai PACE, 1992: 129

$1 \sigma^{\top}$ e 1 ㅇ , China: Hainan, Jiaxi, 480 m, 14.VI.1999, leg. J.R. Fellowes.

\section{Distribuzione:}

Specie finora nota del Nepal.

\section{Drusilla Leach, 1819}

\section{Drusilla philippina (Bernhauer, 1915), comb. n.}

Astilbus philippinus Bernhauer, 1915: 125

1 ㅇ, China: Hainan, Jiaxi, 480 m, 14.VI.1999, leg. J.R. Fellowes.

\section{Distribuzione:}

Specie finora nota delle Filippine.

Nota:

Esemplare da me confrontato con l'holotypus o (Museo di Chicago).

\section{Drusilla gibberella PACE, 1998}

Drusilla gibberella PACE, 1998: 966

1 , China: Hainan, Ba Wang Ling N.R., 1000 m, IV.1998, leg. J.R. Fellowes.

\section{Distribuzione:}

Specie già nota dell'Hainan e Hong Kong. 


\section{Drusilla yunnanensis PACE, 1993}

Drusilla yunnanensis PACE, 1993: 112

1 ㅇ, China: N-Yunnan, Dali Bai Nat. Aut. Pref., Diancang Shan, $1 \mathrm{~km} \mathrm{~W} \mathrm{Dali,} \mathrm{foot} \mathrm{hill} \mathrm{of}$

Diancang Shan, $25^{\circ} 41.9^{\prime} \mathrm{N} 100^{\circ} 08.4^{\prime} \mathrm{E}, 2170 \mathrm{~m}$, 28.VIII.2003, leg. A. Smetana.

\section{Distribuzione:}

Specie già nota dello Yunnan, Hubei e Hunan.

\section{Drusilla damingensis n. sp.}

(Figg. 22-25)

\section{Materiale tipico:}

Holotypus ơ, China: Guangxi, Da Ming Shan, 1000 m, 13.V.1997, leg. J. Fellowes.

Paratypus: 1 , stessa provenienza (IRSNB).

\section{Descrizione:}

Lunghezza 4,8 mm. Corpo lucido, pronoto ed elitre opachi. Corpo nero-bruno, capo e pronoto bruni, antenne brune con i quattro antennomeri basali giallo-rossicci, zampe giallo-rossicce con terzo o metà posteriore dei femori bruno-rossicci. Secondo antennomero molto più corto del primo, terzo molto più lungo del secondo, quarto a decimo più lunghi che larghi. Occhi lunghi quanto le tempie. Reticolazione del capo superficiale, quella di pronoto ed elitre assente, quella del sesto urotergo libero del $\sigma^{*}$ fortissima. Margine posteriore del sesto urotergo libero del $\sigma^{*}$ crenellato e arcuato. Punteggiatura del capo superficiale, quella delle elitre fittissima e profonda. Granulosità del pronoto saliente e poco fitta sul terzo anteriore, sul resto del pronoto granulosità fitta e saliente. Tre uroterghi liberi basali con granulosità saliente. Disco del capo profondamente concavo. Edeago figg. 23-24, spermateca fig. 25.

\section{Comparazioni:}

Per la forma dell'edeago e del pronoto, la nuova specie è simile a D. ganglbaueri (BERnHAuer, 1906) del Turkmenistan e Tagikistan. Se ne distingue per l'edeago più stretto, in visione laterale, e a lati preapicali più arcuati, in visione ventrale. L'introflessione apicale del bulbo distale della spermateca è larghissima nella nuova specie, stretta in ganglbaueri. Gli antennomeri sesto a nono sono più lunghi che larghi nella nuova specie, lunghi quanto larghi in ganglbaueri.

\section{Etimologia:}

La nuova specie prende nome dal Monte Da Ming. Shan significa monte.

\section{Drusilla cenwanglaomontis $\mathbf{n}$. sp.}

(Figg. 2 e 26)

\section{Materiale tipico:}

Holotypus + , China: Guangxi, Cenwanglaoshan, 1350 m, 31.VII.1999, leaf litter, leg. J.R. Fellow (IRSNB). 


\section{Descrizione:}

Lunghezza 4,2 $\mathrm{mm}$. Corpo lucido e giallo-rossiccio, quarto urotergo libero bruno, antenne brune con i tre antennomeri basali e l'undicesimo giallo-rossicci, zampe giallo-rossicci con parte distale dei femori posteriori giallo-bruna. Secondo antennomero più corto del primo, terzo più lungo del secondo, quarto a decimo più lunghi che larghi. Occhi lunghi quanto le tempie. Reticolazione del corpo assente. Granulosità del capo poco evidente, quella della metà anteriore del pronoto saliente. Punteggiatura della metà posteriore del pronoto forte, quella delle elitre fittissima e profonda, ma diradata lungo il margine posteriore. Pronoto con profondo e larga depressione mediana. Spermateca fig. 26.

\section{Comparazioni:}

Per la forma della spermateca la nuova specie è simile a $D$. obliqua (Bernhauer, 1916) della Malaysia, di cui ho esaminato la serie tipica (Museo di Chicago). Se ne distingue per la profonda introflessione apicale del bulbo distale della spermateca e per la parte prossimale della stessa spermateca lunga e ampiamente ricurva (introflessione apicale del bulbo distale della spermateca corte e parte prossimale appena ricurva in obliqua).

\section{Etimologia:}

La nuova specie prende nome dal toponimo Cenwanglaoshan. Shan significa monte.

\section{Drusilla gilva n. sp.}

(Figg. 3 e 27-28)

\section{Materiale tipico:}

Holotypus + , China, Sichuan, Qingcheng Shan, 65 km NW Chengdu, 3053'N 10333'E, 8001000 m, 18.V/3-4.VI.1999 m, 8 km W Taiping. VI.1997, leg. A. Pütz (SMTD).

\section{Descrizione:}

Lunghezza 4,2 mm. Corpo lucido e giallo-rossiccio, due terzi posteriori delle elitre e terzo urotergo libero rossicci, quarto urotergo libero bruno, antenne giallo-rossicce, zampe gialle. Secondo antennomero più corto del primo, terzo più lungo del secondo, quarto lungo quanto largo, quinto a decimo trasversi. Occhi lunghi quanto le tempie. Reticolazione del corpo assente. Punteggiatura del capo molto superficiale, assente sulla fascia longitudinale mediana. Granulosità di pronoto ed elitre saliente a raspa. Disco del capo appiattito. Pronoto con profondo solco mediano, confluente in una fossetta posteriore. Uroterghi liberi nudi. Spermateca fig. 27, sesto urotergo libero della + fig. 28.

\section{Comparazioni:}

Per la forma della spermateca, la nuova specie è simile a D. ganglbaueri (Bernhauer, 1906) dell'Asia Centrale. Se ne distingue per la parte prossimale della spermateca esile in rapporto alla parte distale e per il margine posteriore del sesto urotergo libero della $\odot$ con sei corti lobi (con numerosi cortissimi lobi esili in ganglbaueri).

\section{Etimologia:}

La nuova specie deriva il suo nome dal latino gilvus, giallo. 


\section{Drusilla sinicola n. sp.}

(Figg. 29-30)

\section{Materiale tipico:}

Holotypus + , China: Sichuan, Gongga Shan, Hailougou for. above camp 2, 2800 m, 5.VIII.1998, leg. A. Smetana (MHNG).

\section{Descrizione:}

Lunghezza 6,6 mm. Avancorpo opaco, addome lucido. Corpo nero, addome rossiccio, antenne giallo-rossicce con antennomero basale bruno, zampe rossicce. Secondo antennomero più corto del primo, terzo più lungo del secondo, quarto a decimo più lunghi che larghi. Occhi molto più corti delle tempie. Reticolazione di capo e pronoto forte, quella delle elitre superficiale, quella dell'addome assente. Granulosità di capo ed elitre fittissima e poco evidente. Punteggiatura del pronoto fittissima e poco profonda. Carena marginale posteriore delle elitre che risale fino alla parte posteriore della sutura delle stesse elitre. Punti mediani forti e profondi nel fondo dei solchi trasversi basali dei quattro uroterghi liberi basali. Spermateca fig. 30 .

\section{Comparazioni:}

Per la forma della spermateca la nuova specie è comparabile con D. ganglbaueri (Bernhauer, 1906) dell'Asia Centrale. Se ne distingue per la forte riduzione oculare e per la cortissima parte prossimale della spermateca (assai lunga in ganglbaueri).

\section{Etimologia:}

La nuova specie ha nome che significa «Abitatrice della Cina».

\section{Diplopleurus Bernhauer, 1915}

\section{Diplopleurus cooteri PACE, 1998}

Diplopleurus cooteri PACE, 1998: 968

1 ㅇ, China: Sichuan, Qingcheng Shan, 1000-1300 m, 18-20.VI.1995, leg. Heinz.

\section{Distribuzione:}

Specie finora nota dello Zhejiang.

\section{Zyras STEPHENS, 1835}

\section{Zyras (Zyras) wei PACE, 1993}

Zyras (Zyras) wei PACE, 1993: 114

4 es., China: Sichuan, Gongga Shan, Hailougou, above camp III, ca. 3200 m, 7.VII.1996, leg. A. Smetana; 1 \% , China: W-Sichuan, pass btw. Zhanla and Nanping, 4000 m, VI.1996, leg. M. Häckel; 1 ㅇ, China: N-Yunnan, Dali Bai Nat. Aut. Pref., Diancang Shan, $3 \mathrm{~km}$ W Dali, $25^{\circ} 41.9^{\prime} \mathrm{N} 100^{\circ} 08.4^{\prime} \mathrm{E}$, 3.IX.2003, leg. A. Smetana; $20^{\top} 0^{\star}$, China: W-Hubei, Daba Shan pass

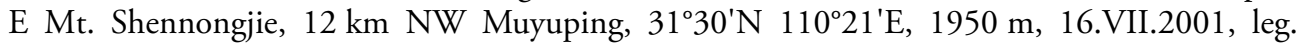
A. Smetana; 1 es., China: S-Shaanxi, Qin Ling Shan, mountain range W pass on rd. Xi'an, 
Shagoujie, 45 km SSW Xi'an, 2675 m, 3352'N 10846'E, 25.VII.2001, leg. A. Smetana; 1 es., China: N. Yunnan, Lijiang, Naxi Aut. Co., E Yulongxue Shan, $30 \mathrm{~km} \mathrm{~N} \mathrm{Lijiang,} 27^{\circ} 09.0^{\prime} \mathrm{N}$ $100^{\circ} 14.9^{\prime} \mathrm{E}, 2800-2900 \mathrm{~m}, 13 . \mathrm{VIII} .2003$, leg. A. Smetana.

\section{Distribuzione:}

Specie finora nota del Guizhou.

\section{Zyras (Zyras) songanus PACE, 1993}

Zyras (Zyras) songanus PACE, 1993: 114

$10^{\star}$, China: W-Hubei, Daba Shan mtn. range NE Muyuping, creek valley, $4 \mathrm{~km}$ N Muyuping, 1700 m, 21.VII.2001, leg. A. Smetana; 1 es., China: W-Hubei, Daba Shan pass E of Mt. Da Shennongjia, $12 \mathrm{~km}$ NW Muyuping, 3130'N 110²1'E, 19.VII.2001, $2050 \mathrm{~m}$, leg. A. Smetana; 3 es., China: S-Shaanxi, Qin Ling Shan, mountain range W pass on rd. Xi'an, Shagoujie, $45 \mathrm{~km}$ SSW Xi'an, 2675 m, 335' $\mathrm{N} 108^{\circ} 46^{\prime} \mathrm{E}, 25 . V I I .2001$, leg. A. Smetana; 1 es., China: N.Yunnan, Lijiang, Naxi Aut. Co., E Yulongxue Shan, $30 \mathrm{~km} \mathrm{~N} \mathrm{Lijiang,} 27^{\circ} 09.0^{\prime} \mathrm{N} 100^{\circ} 14.9^{\prime} \mathrm{E}, 2800$ 2900 m, 13.VIII.2003, leg. A. Smetana.

\section{Distribuzione:}

Specie finora nota di Beijing e Shaanxi.

\section{Zyras (Zyras) restitutus PACE, 1993}

Zyras (Zyras) restitutus PACE, 1993: 114

1 o $^{\star}$, China: Shaanxi, Nanwutaishan, 4.IV.2003, leg. G. de Rougemont.

\section{Distribuzione:}

Specie già nota del Sichuan.

\section{Zyras (Zyras) shaanxiensis PACE, 1998}

Zyras (Zyras) shaanxiensis PACE, 1998: 971

11 es., China: Sichuan, Emei Shan, 3000 m, 17.VII.1996, leg. A. Smetana.

\section{Distribuzione:}

Specie finora nota dello Shaanxi.

\section{Zyras (Zyras) alboantennatus PACE, 1984}

Zyras (s. str.) alboantennatus PACE, 1984: 460

1 đ̆$^{\star}$, China: Yunnan, Lijiang, Yushuizhai, ca. 2600 m, 14.IV.2003, leg. G. de Rougemont.

\section{Distribuzione:}

Specie finora nota della Birmania. 


\section{Zyras (Zyras) qingchengensis n. sp.}

(Figg. 4 e 31-33)

\section{Materiale tipico:}

Holotypus $0^{\star}$, China, Sichuan, Wennjian Distr., Guanxian Co, Qingcheng Shan, $65 \mathrm{~km}$ NW Chengdu, 30 $53^{\prime} \mathrm{N} 103^{\circ} 32^{\prime} \mathrm{E}, 800-1000 \mathrm{~m}, 8 \mathrm{~km}$ W Taiping, 18.V/3-4.VI.1997, leg. A. Pütz (SMTD).

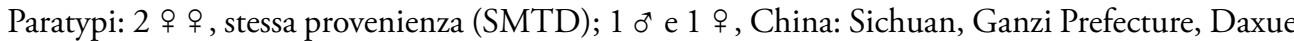
Shan, N Kanding, $30^{\circ} 03^{\prime}$ N, $101^{\circ} 57^{\prime} E, 2600-2700$ m, 22-24.V.1997, leg. A. Pütz (SMTD).

\section{Descrizione:}

Lunghezza 6,9 mm. Corpo lucido e nero-bruno, metà basale delle elitre rossiccia, margine posteriore dei quattro uroterghi liberi basali rossiccio, antenne brune con i due antennomeri basali e base del terzo giallo-rossicci, zampe giallo-rossicce. Secondo antennomero più corto del primo, terzo più lungo del secondo, quarto lungo quanto largo, quinto e sesto più lunghi che larghi, settimo a decimo debolmente trasversi. Occhi più corti delle tempie. Reticolazione del corpo assente. Punteggiatura di capo e pronoto forte, ma assente su una stretta fascia longitudinale mediana del capo e sui due terzi anteriori del pronoto. Punteggiatura delle elitre profonda e fitta, quella dell'addome confinata nel fondo del solco trasverso basale dei tre uroterghi liberi basali. Edeago figg. 31-32, spermateca fig. 33.

\section{Comparazioni:}

Per il pronoto nettamente punteggiato e per la forma dell'edeago, la nuova specie è simile a Z. beijingensis PACE, 1993 di Pechino. Se ne distingue per il pronoto più trasverso con rapporto larghezza/lunghezza pari a 1,2 (rapporto larghezza/lunghezza pari a 1,05 in beijingensis). L'edeago della nuova specie ha profilo ventrale angoloso presso la "crista apicalis», mentre in beijingensis il profilo ventrale presso la «crista apicalis» è ampiamente ricurvo.

\section{Etimologia:}

La nuova specie prende nome dal Monte Qingcheng.

\section{Zyras (Zyras) sichuanorum n. sp.}

(Figg. 5 e 34-36)

\section{Materiale tipico:}

Holotypus $0^{\star}$, China, Sichuan, Wennjian Distr., Guanxian Co, Qingcheng Shan, $65 \mathrm{~km}$ NW Chengdu, 30 $53^{\circ} \mathrm{N} 103^{\circ} 32^{\prime} \mathrm{E}, 800-1000 \mathrm{~m}, 8 \mathrm{~km}$ W Taiping, 18.V/3-4.VI.1997, leg. A. Pütz (SMTD).

Paratypi: 2 ㅇ ㅇ, China, Sichuan, Wennjian Distr., Guanxian Co, Qingcheng Shan, 56 km NW Chengdu, 3053.84'N 10332.80'E, 975 m, 18.VI.1999, 8 km W Taiping.VI.1997, leg. A. Pütz (SMTD).

\section{Descrizione:}

Lunghezza 7,2 mm. Corpo lucido e giallo-rossiccio, capo, metà posteriore delle elitre e uroterghi liberi quarto e quinto e base del sesto bruni, antenne brune con antennomeri nono a undicesimo giallo pallido, zampe giallo-rossicce. Secondo antennomero più corto del primo, terzo più lungo del secondo, quarto a settimo più lunghi che larghi, ottavo lungo quanto largo, nono e decimo trasversi. 
Occhi lunghi quanto le tempie. Reticolazione del corpo assente. Punteggiatura di capo e pronoto assente, quella delle elitre fitta ed evidente, quella della base degli uroterghi liberi quarto e quinto forte. Pronoto con profondo fossetta mediana posteriore. Edeago figg. 34-35, spermateca fig. 36.

\section{Comparazioni:}

Per la forma dell'edeago, la nuova specie è simile a Z. chinkiangensis Bernhauer, 1939 del NW della Cina, di cui ho esaminato l'holotypus ơ (Museo di Chicago). Se ne distingue per le elitre bicolori (uniformemente bruno-rossicce in chinkiangensis) e per l'edeago strettamente ricurvo al lato ventrale, con sporgenza ventrale stretta (ampiamente ricurvo e con larga sporgenza ventrale in chinkiangensis).

\section{Etimologia:}

La nuova specie ha nome che significa «Dei Sichuanesi».

\section{Zyras (Zyras) yongshengensis n. sp.}

(Figg. 6 e 37-38)

\section{Materiale tipico:}

Holotypus ơ, China: N.Yunnan, Lijiang, Naxi Co., 3 km NW Yongsheng, 53 km WSW Lijiang, $26^{\circ} 41.8^{\prime} \mathrm{N} 100^{\circ} 43.1^{\prime} \mathrm{E}, 1990 \mathrm{~m}, 14 . \mathrm{VIII} .2003$, leg. A. Smetana (SDEI).

\section{Descrizione:}

Lunghezza 5,1 mm. Corpo lucido e giallo-rossiccio, capo elitre e uroterghi liberi quinto e sesto bruni, antenne bruno-rossicce (incomplete), zampe gialle con terzo distale dei femori brunorossiccio. Secondo antennomero più corto del primo, terzo più lungo del secondo, quarto a sesto più lunghi che larghi, antennomeri restanti perduti. Occhi più corti delle tempie. Reticolazione del corpo assente. Punteggiatura del capo assente, quella del pronoto fine e superficiale, quella delle elitre forte, più fitta alla base che sulla parte posteriore. Pronoto con fossetta mediana basale. Elitre più corte del pronoto. Edeago fig. 37-38.

\section{Comparazioni:}

A motivo delle elitre più corte del pronoto e per le ali ridotte, non atte al volo, la nuova specie è nettamente distinta dalle specie cinesi note. L'edeago della nuova specie è simile a quello di $Z$. chinkiangensis Bernhauer, 1939 del NW della Cina, di cui ho esaminato l'holotypus ơ (Museo di Chicago). Se ne distingue per l'apice dell'edeago ottuso, in visione ventrale, (apice acuto in chinkiangensis) e per la sua minore lunghezza, 0,67 $\mathrm{mm}(1,02 \mathrm{~mm}$ di lunghezza dell'edeago di chinkiangensis).

\section{Etimologia:}

La nuova specie prende nome dal toponimo Yongsheng.

\section{Zyras (Rhynchodonia) rufithorax CAMERON, 1930}

Zyras rufithorax CAMERon, 1930: 158

Drusilla hanoiensis PAŚNIK, 2000: 1058, syn. n.

1 ㅇ, China: SE-Hainan, Diaoluoshan, 320 m, 29.V.1999, leg. J.R. Fellowes. 


\section{Distribuzione:}

Specie finora nota della Malaysia, Vietnam, Thailandia.

Nota:

Specie determinata in base al mio esame della serie tipica di $20^{\star} o^{\star}$ e 5 q $q$ etichettata Malay Peninsula nr. Jitra, Catchement Area, 8-10. April 1928, Zyras rufithorax CAM., Type (Museo di Londra). L'inconfondibile forma della spermateca di questa specie permette di riconoscere la nuova sinonimia.

\section{Zyras (Rhynchodonia) diaoluomontis n. sp.}

(Figg. 7 e 39-40)

\section{Materiale tipico:}

Holotypus ơ , China: SE-Hainan, Diaoluoshan, 350 m, 27.V.1999, leg. J.R. Fellowes (IRSNB).

\section{Descrizione:}

Lunghezza $6 \mathrm{~mm}$. Corpo lucido e nero-bruno, elitre rossicce, margine posteriore dei quattro uroterghi liberi basali bruno-rossicci, antenne brune con i tre antennomeri basali giallo-rossicci, zampe giallo-rossicce. Secondo antennomero molto più corto del primo, terzo più corto del secondo, quarto a decimo fortemente trasversi. Occhi lunghi quanto le tempie. Reticolazione del capo forte sul disco, superficiale sul resto della sua superficie, quella del pronoto evidente sulla fascia longitudinale mediana, superficiale ai lati di essa, quella delle elitre evanescente. Punteggiatura del capo superficiale, quella del pronoto forte, ma distribuita su due file mediane e ai lati, quella delle elitre evidente e irregolarmente distribuita, quella dell'addome ben distinta. Quinto urotergo libero del $\sigma^{*}$ con stretto tubercolo mediano al margine posteriore, margine posteriore del sesto urotergo libero del ơ bisinuato. Edeago figg. 39-40.

\section{Comparazioni:}

Per la forma del corpo e delle antenne, la nuova specie è simile a $Z$. atricillus PACE, 1984 della Birmania. Se ne distingue per la presenza di reticolazione sulla fascia longitudinale mediana del pronoto (fascia longitudinale mediana del pronoto di atricillus senza reticolazione) e per gli antennomeri quarto a decimo fortemente trasversi (poco trasversi in atricillus). Non è noto il $\sigma^{*}$ di atricillus.

\section{Etimologia:}

La nuova specie ha nome che significa «Del Monte Diaoluo». Shan significa monte.

\section{Zyras (Rhynchodonia) fellowesi n. sp.}

(Figg. 41-43)

\section{Materiale tipico:}

Holotypus ठ, China: Guandong Pr., Hri Shi Ding, 24.IV.1997, leg. J. Fellowes (IRSNB).

\section{Descrizione:}

Lunghezza 12,5 mm. Corpo lucido, tranne il capo opaco. Corpo bruno-rossiccio, capo bruno, antenne bruno-rossicce, zampe rossicce con ginocchia bruno-rossicce. Secondo antennomero 
molto più corto del primo, terzo più corto del secondo, quarto a decimo lunghi quanto larghi. Occhi più lunghi delle tempie. Reticolazione del capo forte, assente sulle tempie, quella di pronoto ed elitre assente, quella dell'addome superficiale. Punteggiatura del capo evidente, quella del pronoto ombelicata e profonda, quella delle elitre assai profonda e fitta, quella dell'addome evidente e fine. Pronoto con solco mediano sui tre quarti posteriori. Secondo urotergo libero del $\sigma^{\star}$ prolungato all'indietro da due lunghi lobi triangolari, terzo con un rilievo mediano e due dentini laterali al margine posteriore. Quinto urotergo libero del $\sigma^{t}$ con depressioni laterali longitudinali e un rilievo mediano posteriore triangolare. Edeago figg. 42-43.

\section{Comparazioni:}

La nuova specie è simile a $Z$. rougemonti PACE, 1984 della Birmania. Se ne distingue per le appendici preapicali dell'armatura genitale interna dell'edeago sporgenti molto larghe, $0,013 \mathrm{~mm}$, (molto strette in rougemonti, di 0,004 mm), per l'apice dell'edeago tronco, in visione ventrale, (inciso in rougemonti) e per le appendici del secondo urotergo libero del $\sigma^{*}$ larghe (strette in rougemonti).

\section{Etimologia:}

La nuova specie è dedicata al suo raccoglitore.

\section{Pedinopleurus Cameron, 1939}

\section{Pedinopleurus jiuwanmontis n. sp.}

(Figg. 8 e 44-45)

\section{Materiale tipico:}

Holotypus ${ }^{\star}$, China, Guangxi, Jiuwanshan, 25.VII.1998, leg. R. Fellowes (IRSNB).

\section{Descrizione:}

Lunghezza 4,8 mm. Corpo lucido e giallo-rossiccio, capo nero-bruno, elitre rossicce, uroterghi liberi quarto e quinto bruni, antenne brune con i tre antennomeri basali giallo-rossicci, zampe giallo-rossicce. Secondo antennomero molto più corto del primo, terzo più corto del secondo, quarto a decimo fortemente trasversi. Occhi più lunghi delle tempie. Reticolazione del corpo assente. Punteggiatura di capo e addome superficiale, quella del pronoto evidente, quella delle elitre forte e fitta.

Fine e debole solco mediano del pronoto. Primo urotergo libero del $o^{\star}$ con lunga spina mediana sporgente dal margine posteriore. Edeago figg. 44-45.

\section{Comparazioni:}

Per la forma dell'edeago e del corpo, la nuova specie è simile a P. spinibasis PACE, 2000 della Thailandia. Se ne distingue per l'edeago ampiamente arcuata al lato ventrale (non arcuato in spinibasis) e per l'armatura genitale interna dell'edeago esile e ridotta (armatura genitale interna dell'edeago forte e composta di numerosi pezzi in spinibasis). Il pronoto della nuova specie è giallo-rossiccio, bruno con lati rossicci in spinibasis.

\section{Etimologia:}

La nuova specie prende nome dal Monte Jiuwan. 
Thamiaraeini Fenyes, 1921

Eutaenoglossa PACE, 1984

Eutaenoglossa schuelkei $\mathrm{n} . \mathrm{sp}$.

(Figg. 9 e 46)

\section{Materiale tipico:}

Holotypus + , China: W-Sichuan, Ya’an Prefecture, Fulin Co., Daxiang Ling, road HanyuanjieSiping, $51 \mathrm{~km}$ NNE Shimian, smal stream, $2300 \mathrm{~m}, 10 . \mathrm{VII} .1999,29^{\circ} 39^{\prime} \mathrm{N} 102^{\circ} 37^{\prime} \mathrm{E}$, leg. D.W. Wrase (MNHU).

\section{Descrizione:}

Lunghezza $2 \mathrm{~mm}$. Corpo lucido e bruno-rossiccio, uroterghi liberi quarto e quinto bruni, antenne bruno-rossicce con apice distale dell'undicesimo giallo-rossiccio, zampe giallo-rossicce. Secondo antennomero più corto del primo, terzo più corto del secondo, quarto più lungo che largo, quinto lungo quanto largo, antennomeri sesto a decimo trasversi. Occhi più lunghi delle tempie. Reticolazione del corpo assente. Punteggiatura di capo e pronoto molto superficiale, quella delle elitre fitta e forte. Granulosità dell'addome saliente. Assenza di scultura embricata sugli uroterghi liberi. Spermateca fig. 46.

\section{Comparazioni:}

La spermateca della nuova specie è simile a quella di E. nigra PACE, 1984 del Nepal. Se ne distingue per gli occhi più lunghi delle tempie (occhi ridotti, molto più corti delle tempie in nigra) e per il colore bruno-rossiccio del corpo e antenne (nero profondo, antenne comprese in nigra).

\section{Etimologia:}

La nuova specie è dedicata a Michael Schülke di Berlino per avermi affidato in studio parte delle Aleocharinae da lui raccolte in Cina.

\section{Platorischna PACE, 1991}

\section{Platorischna vietnamensis PACE, 1992}

Platorischna vietnamensis PACE, 1992: 128

2 우 오, China: Guangxi, Dawangling, 1420 m, 5.VIII.1999, leg. J. Fellowes; 1 우, China: Guangxi, Cenwanglaoshan, 1350 m, 31.VII.1999, leg. J.R. Fellow; $1 \sigma^{\star}$, China: Guangxi, Huaping, 19.VIII.1998, leg. J.R. Fellow.

\section{Distribuzione:}

Specie finora nota solo del Vietnam. 


\section{Platorischna sinensis n. sp.}

(Figg. 10 e 47-50)

\section{Materiale tipico:}

Holotypus 9 , China, Sichuan, Qingcheng Shan, 65 km NW Chengdu, 3053'N 10333'E, 8001000 m, 18.V/3-4.VI.1999 m, 8 km W Taiping.VI.1997, leg. A. Pütz (SMTD).

Paratypi: 11 es., stessa provenienza (SMTD).

\section{Descrizione:}

Lunghezza 1,6 mm. Corpo lucido e giallo-rossiccio, due terzi distali delle elitre, uroterghi liberi quarto e base del quinto bruni, antenne brune con i tre antennomeri basali gialli, zampe gialle. Secondo antennomero più corto del primo, terzo più corto del secondo, quarto a decimo trasversi. Occhi più corti delle tempie. Reticolazione del corpo assente. Granulosità dell'avancorpo fine e saliente, quella dell'addome fitta, assai fine e superficiale. Edeago figg. 47-48, spermateca fig. 49.

\section{Comparazioni:}

Per la forma della spermateca la nuova specie è più simile a $P$. nepalensis PACE, 1991 del Nepal che a P. vietnamensis PACE, 1993 del Vietnam. L'introflessione apicale del bulbo distale della spermateca della nuova specie è esile e non enorme come in nepalensis e vietnamensis, e la parte prossimale della stessa spermateca è sottile e lunga nella nuova specie (corta o cortissima o larga nelle due specie a confronto).

\section{Etimologia:}

La nuova specie ha nome di derivazione latina che significa «Cinese».

\section{Platorischna sichuanensis n. sp.}

(Figg. 11 e 51)

\section{Materiale tipico:}

Holotypus + , China: W-Sichuan, Ya'an Prefecture, Tianquan Co., Jiaajin Shan, valley above Labahe nr. Station, $57 \mathrm{~km} \mathrm{~W}$ Ya’an, $1800 \mathrm{~m}, 30^{\circ} 08^{\prime} 63^{\prime \prime} \mathrm{N} \mathrm{102} 25^{\prime} 18^{\prime \prime} \mathrm{E}, 12 . V I I .1999$, light forest, leg. A. Pütz (SMTD).

Paratypus: 1 \% , stessa provenienza (SMTD).

\section{Descrizione:}

Lunghezza 1,6 mm. Corpo lucido e giallo-bruno, antenne brune con i tre antennomeri basali gialli, zampe gialle. Secondo antennomero lungo quanto il primo, terzo più corto del secondo, quarto a decimo fortemente trasversi. Occhi molto più corti delle tempie. Reticolazione del corpo assente. Granulosità dell'avancorpo fitta, fine e saliente, quella dell'addome fitta, superficiale e rada sul quinto urotergo libero. Spermateca fig. 51.

\section{Comparazioni:}

Per la forma della spermateca la nuova specie non è comparabile con specie note. La spermateca della nuova specie, infatti, è ridotta al bulbo distale, essendo la parte prossimale e intermedia ridottissime.

\section{Etimologia:}

La nuova specie prende nome dal Sichuan. 
Lasiokoryphina n. gen.

(Figg. 12 e 52-55)

\section{Diagnosi:}

Per l'habitus e forma della spermateca, il nuovo genere è affine al genere Platorischna PACE, 1991. La ligula, tuttavia, ha forma molto differente, fig. 53 , vale a dire non divisa in due rami apicali come in genere Platorischna, fig. 50, ma intera con due setole preapicali.

\section{Descrizione:}

Labbro trasverso, arrotondato agli angoli anteriori, debolmente smarginato al margine anteriore. Mandibole prominenti; la mandibola destra con un piccolo dente o con una smarginatura solamente a metà del margine interno. Maxillae con il lobo esterno pubescente e membranoso all'apice; lobo interno ricurvo verso l'interno alla punta distale, con spine apicali lunghe, con setole sottili sulla porzione basale e membranosa. Palpi mascellari 4-articolati, moderatamente lunghi, fig. 54; terzo articolo più lungo e più largo del secondo; quarto corto e lesiniforme, fig. 54. Mento trapezoidale, debolmente smarginato al margine anteriore. Ligula stretta e intera, lunga quanto il primo articolo dei palpi labiali, fig. 53. Palpi labiali di due articoli; articolo secondo più corto e più stretto del primo. Antenne di undici articoli; i primi tre articoli più lunghi; articoli terzo più corto del secondo, antennomeri quarto a decimo trasversi, undicesimo lungo quasi quanto $i$ due articoli precedenti riuniti. Capo prominente con tempie lievemente divergenti all'indietro, collo largo; occhi più corti delle tempie; parte inferiore delle tempie non marginata. Pronoto traverso, più stretto delle elitre, non sinuato davanti agli angoli posteriori esterni. Elitre debolmente smarginate presso gli angoli posteriori esterni. Addome poco ristretto all'indietro, solo il primo urotergo libero solcato trasversalmente alla base. Zampe corte; tarsi 4-5-5 articolati; primo protarsomero, primo mesotarsomero e primo metatarsomero lungo quanto il seguente fig. 55 . Processo mesosternale acuto. Mesocoxe contigue. Spermateca fig. 52.

\section{Typus generis:}

Lasiokoryphina sinica $\mathrm{n}$. sp.

\section{Etimologia:}

Il nome del nuovo genere è composto dai vocaboli in greco antico $\lambda \dot{\alpha} \delta$ เo $=$ lasios $=$ setoloso e корvч $\dot{\eta}=$ koryphe = apice. Significa pertanto «Apice setoloso», in riferimento alle setole inserite ai lati della ligula della nuova specie di appartenenza.

\section{Lasiokoryphina sinica n. sp.}

(Figg. 12 e 52-55)

\section{Materiale tipico:}

Holotypus + , China: Sichuan, Ganzi Prefecture, Daxue Shan, Gongga Shan, Hailougou glacier park, $102^{\circ} 04^{\prime} \mathrm{E} 29^{\circ} 36^{\prime} \mathrm{N}$, river valley, ca. $1 \mathrm{~km}$ above camp I, $2100 \mathrm{~m}, 28-31 . V .1997$, leg. A. Pütz (SMTD).

\section{Descrizione:}

Lunghezza 1,7 mm. Corpo lucido e bruno, antenne brune, zampe giallo-rossicce. Secondo antennomero più lungo del primo, terzo più corto del secondo, quarto a decimo trasversi. Occhi 
più corti delle tempie. Reticolazione di capo e pronoto assente, quella delle elitre superficiale, quella dell'addome poligonale irregolare evanescente. Granulosità di capo e pronoto fitta, fine e molto saliente, quella delle elitre fitta e poco saliente, quella dell'addome fine, fitta e superficiale. Spermateca fig. 52 .

\section{Etimologia:}

La nuova specie ha nome che significa «Cinese».

\section{Medeterusa PACE, 1987}

\section{Medeterusa chinensis n. sp.}

(Figg. 13 e 56-57)

\section{Materiale tipico:}

Holotypus ơ, China: Hainan, Wuzhishan, 880 m, 9.VI.1999, leg. J.R. Fellow (IRSNB).

\section{Descrizione:}

Lunghezza 2,1 mm. Corpo lucido e bruno-rossiccio, addome giallo-rossiccio, antenne brune con i due antennomeri basali giallo-rossicci, zampe giallo-rossicce. Secondo antennomero più corto del primo, terzo più corto del secondo, quarto a decimo trasversi. Occhi più lunghi delle tempie. Reticolazione di capo ed elitre superficiale, quella del pronoto evidente, quella dell'addome assente. Punteggiatura del capo assai poco distinta. Granulosità di pronoto ed elitre evidente, quella dell'addome più fitta alla base che all'indietro dello stesso addome. Edeago figg. 56-57.

\section{Comparazioni:}

Per la forma delle parti boccali, la nuova specie è attribuita al genere Medeterusa PACE, 1987 diffuso in Nepal e Hong Kong. Per la forma dell'edeago è simile a M. smetanai PaCE, 1991 del Nepal. Se ne distingue per l'edeago più lungo, $0,24 \mathrm{~mm}$, di quello di smetanai, lungo $0,16 \mathrm{~mm}$, per essere poco profondamente arcuato al lato ventrale (molto arcuato in smetanai) e per le elitre più lunghe con rapporto lunghezza sutura elitrale/lunghezza pronoto pari a 1,4 (1 in smetanai).

\section{Etimologia:}

La nuova specie ha nome che significa «Cinese».

\section{Mimacrotona Cameron, 1920}

\section{Mimacrotona chinensis n. sp.}

(Figg. 58-60)

\section{Materiale tipico:}

Holotypus ơ, China: Sheun Shui (MNHL).

Paratypi: 1 1 esemplare senza addome, stessa provenienza.

\section{Descrizione:}

Lunghezza 1,6 mm. Corpo lucido e bruno-rossiccio, antenne brune con i tre antennomeri basali rossicci e undicesimo bruno-rossiccio, zampe gialle. Secondo antennomero più corto del primo, 
terzo più corto del secondo, quarto a decimo trasversi. Occhi più corti delle tempie. Reticolazione del corpo assente. Granulosità del capo fine e superficiale, quella di pronoto ed elitre evidente. Addome coperto di scultura embricata evanescente. Edeago figg. 58-59, spermateca fig. 60.

\section{Comparazioni:}

Per la forma dell'edeago la nuova specie è simile a M. rougemonti PACE, 1998 di Hong Kong., ma l'edeago della nuova specie è ampiamente arcuato al lato ventrale (strettamente arcuato in rougemonti), l'armatura genitale interna dell'edeago non presenta un lungo pezzo filiforme come in rougemonti e gli occhi sono più corti delle tempie (occhi più lunghi delle tempie in rougemonti).

\section{Etimologia:}

La nuova specie ovviamente prende nome dalla Cina.

\section{Mimoxypoda Cameron, 1925}

\section{Mimoxypoda chinensis PACE, 1998}

Mimoxypoda chinensis PACE, 1998: 953

$30^{\star} o^{\star}$, China: Border Shaanxi-Sichuan (Daba Shan), pass $20 \mathrm{~km}$ SSE Zhenping, 1700-1800, $31^{\circ} 44^{\prime} \mathrm{N} 109^{\circ} 35^{\prime} \mathrm{E}$, 9.VII.2001, yung dry mixed forest, field edge, small creek valley, moss, sifted, leg. M. Schülke; 6 es., China: W-Hubei, Daba Shan creek valley, 8 km NW Muyuping, $31^{\circ} 29^{\prime} \mathrm{N} 110^{\circ} 22^{\prime} \mathrm{E}, 1540 \mathrm{~m}, 18 . \mathrm{VII} .2001$, leg. Wrase; 1 es., China: W-Hubei (Daba Shan), mountain range, pass $12 \mathrm{~km} \mathrm{~N}$ Muyuping, 31 $32^{\prime} \mathrm{N} 110^{\circ} 26^{\prime} \mathrm{E}, 2380 \mathrm{~m}, 17 . V I I .2001$, moss, leg. M. Schülke.

\section{Distribuzione:}

Specie finora nota dello Yunnan.

\section{Mimoxypoda sinica n. sp.}

(Figg. 14 e 61-63)

\section{Materiale tipico:}

Holotypus ơ, China: W-Sichuan, Ya'an Prefecture, Tianquan Co., Jiajin Shan, valley above Labahe nr., $57 \mathrm{~km}$ W Yaan, $1800 \mathrm{~m}, 30^{\circ} 06^{\prime} \mathrm{N} 102^{\circ} 25^{\prime} \mathrm{E}, 12$.VII.1999, light forest, leg. M. Schülke (MNHU).

Paratypus: 1 ㅇ, stessa provenienza.

\section{Descrizione:}

Lunghezza 1,4 mm. Corpo lucido e giallo-bruno, capo e uroterghi liberi terzo a quinto bruni, antenne rossicce con i due antennomeri basali giallo-rossicci, zampe gialle. Secondo antennomero più corto del primo, terzo più corto del secondo, quarto a decimo fortemente trasversi. Occhi lunghi quanto le tempie. Reticolazione del corpo assente. Granulosità del capo superficiale, quella del pronoto evidente, quella delle elitre saliente, quella dell'addome fitta e assente lungo il margine posteriore di ciascun urotergo libero. Edeago figg. 61-62, spermateca fig. 63. 


\section{Comparazioni:}

Per la forma dell'edeago e della spermateca la nuova specie è simile a $M$. parva CAmeron, 1939 dell'India e Nepal, di cui ho esaminato la serie tipica di $10^{t}$ e 1 ㅇ (Museo di Londra). Se ne distingue per la parte prossimale della spermateca nettamente più larga, $0,027 \mathrm{~mm}$, di quella di parva, 0,013 $\mathrm{mm}$, per la presenza di introflessione apicale del bulbo distale della spermateca, assente in parva e per la «crista apicalis» dell'edeago assai saliente (assente in parva).

\section{Etimologia:}

La nuova specie ha nome che significa «Cinese».

\section{Mimoxypoda sinorum n. sp.}

(Figg. 15 e 64-66)

\section{Materiale tipico:}

Holotypus $0^{\star}$, China: W-Sichuan, Ya'an Prefecture, Tianquan Co., Jiajin Shan, below Labahe

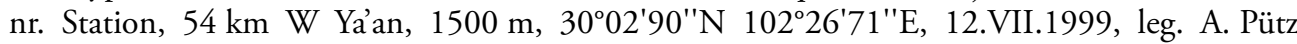
(SMTD).

Paratypi: 4 es., stessa provenienza (SMTD).

\section{Descrizione:}

Lunghezza 1,8 mm. Corpo lucido e giallo-bruno, uroterghi liberi terzo, quarto e base del quinto bruni, antenne brune con i tre antennomeri basali gialli. Secondo antennomero più lungo del primo, terzo più corto del secondo, quarto a decimo fortemente trasversi. Occhi più lunghi delle tempie. Reticolazione del corpo assente. Granulosità di capo e pronoto fitta e superficiale, quella delle elitre saliente, quella dell'addome evanescente, ma saliente sugli uroterghi liberi quarto e quinto. Edeago figg. 64-65, spermateca fig. 66.

\section{Comparazioni:}

Per l'habitus la nuova specie è simile a M. chinensis PACE, 1998 dello Yunnan, ma mentre in chinensis l'edeago è profondamente arcuato al lato ventrale, quello della nuova specie lo è assai poco.

\section{Etimologia:}

La nuova specie ha nome che significa «Dei cinesi».

\section{Litoglossa CAMERon, 1939}

\section{Litoglossa chinensis n. sp.}

(Figg. 16 e 67-69)

\section{Materiale tipico:}

Holotypus $0^{*}$, China: W-Hubei, Daba Shan pass E of Mt. Da Shennongjia, $12 \mathrm{~km} \mathrm{NW}$ Muyuping, 19.VII.2001, 1950 m, leg. A. Smetana (SDEI).

Paratypi: 2 ㅇ , stessa provenienza. 


\section{Descrizione:}

Lunghezza 1,6 mm. Avancorpo debolmente opaco, addome lucido. Corpo nero-bruno, antenne brune, zampe gialle. Secondo antennomero lungo quanto il primo, terzo più corto del secondo, quarto a decimo trasversi. Occhi lunghi quanto le tempie. Reticolazione del corpo assente. Granulosità dell'avancorpo fine, fitta e molto saliente, quella dell'addome fitta ed evidente. Edeago figg. 67-68, spermateca fig. 69.

\section{Comparazioni:}

Per la forma della spermateca la nuova specie è simile a L. opaca CAMEron dell'India, di cui ho esaminato esemplari della serie tipica. Ma la spermateca della nuova specie è più lunga, $0,114 \mathrm{~mm}$, di quella di opaca, 0,071 $\mathrm{mm}$. Non è noto l'edeago di opaca.

\section{Etimologia:}

La nuova specie ovviamente prende nome dalla Cina.

\section{Ringraziamenti}

Per avermi affidato in studio il materiale oggetto del presente lavoro rivolgo i miei più cordiali ringraziamenti ai colleghi Dr. Aleš Smetana di Ottawa, Guillaume de Rougemont di London, Michael Schülke di Berlino e Olaf Jäger dello Senckenberg Museum für Tierkunde di Dresda. Per il prestito di tipi ringrazio il Dr. P. M. Hammond del Museo di Storia Naturale di Londra, il Dr. Didier Drugmand de l'Institut royal des Sciences naturelles de Belgique, il Dr. Lothar Zerche del SDEI di Müncheberg, il Dr A. Newton del Field Museum of Natural History di Chicago, il Dr. Piermauro Giachino del Museo Regionale di Storia Naturale di Torino e il Dr. Manfred Uhlig del Museo Zoologico dell'Università Humboldt di Berlino.

\section{Bibliografia}

Bernhauer, M. 1906: Neue Staphyliniden der paläarktischen Fauna nebst synonymischen Bemerkungen. - Münchener Koleopterologische Zeitschrift 3: 123-128.

Bernhauer, M. 1915a: Zur Staphyliniden-fauna des tropischen Afrika. - Annales Musei Nationalis Hungarici 13: 95-189.

Bernhauer, M. 1915b: Neue Staphyliniden aus Java und Sumatra. - Tijdschrift voor Entomologie 58: 213-243.

Bernhauer, M. 1915c: Zur Staphylinidenfauna der Philippinen: VI. Beitrag zur Kenntnis der indo-malayischen Fauna. - The Philippine Journal of Science 10: 117-129.

Bernhauer, M. 1916: Neue Staphyliniden des indo-malaiischen Faunengebietes, besonders der Philippinen. - Verhandlungen der Kaiserlich-Königlichen Zoologisch-Botanischen Gesellschaft in Wien 66: 418-431.

Bernhauer, M. 1939: Zur Staphylinidenfauna von China und Japan. - Entomologisches Nachrichtenblatt. Troppau. 12 (2) (1938): 97-109.

Cameron, M. 1920: New Species of Staphylinidae from Singapore. - Transactions of the Entomological Society of London 1920: 212-284.

Cameron, M. 1925: Descriptions of new species of Oriental Staphylinidae. - Annali del Museo civico di Storia naturale di Genova 52: 34-49.

Cameron, M. 1930: VIII. New Staphylinidae from the Malay Peninsula (Second Paper). - Journal of the Federated Malay States Museums 16: 154-159.

Cameron, M. 1939: The Fauna of British India, including Ceylon and Burma. Coleoptera, Staphylinidae. Vol. IV: 410 pp. London, Taylor and Francis. 


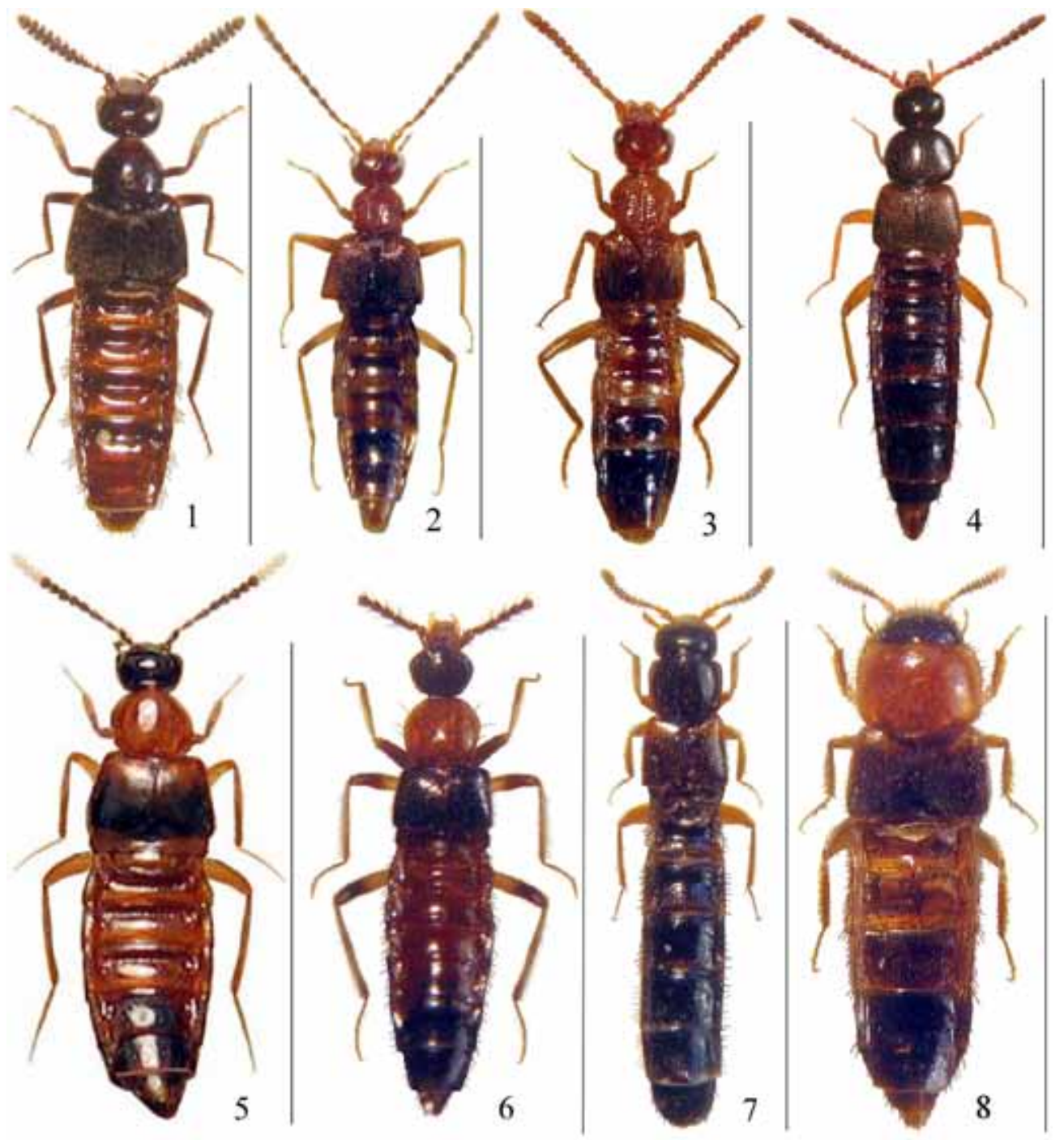

Figg. 1-8: Habitus. 1. Tetrabothrus chinensis $\mathrm{n}$. sp., scala $5,5 \mathrm{~mm}$; 2. Drusilla cenwanglaomontis $\mathrm{n}$. sp., scala 4,2 mm; 3. Drusilla gilva n. sp., scala 4,2 mm; 4. Zyras (Zyras) qingchengensis n. sp., scala 6,9 mm; 5. Zyras (Zyras) sichuanorum n. sp., scala 7,2 mm; 6. Zyras (Zyras) yongshengensis n. sp., scala 5,1 mm; 7. Zyras (Rhynchodonia) diaoluomontis $\mathrm{n}$. sp., scala $6 \mathrm{~mm} ; 8$. Pedinopleurus jiuwanmontis $\mathrm{n}$. sp., scala 4,8 mm. 


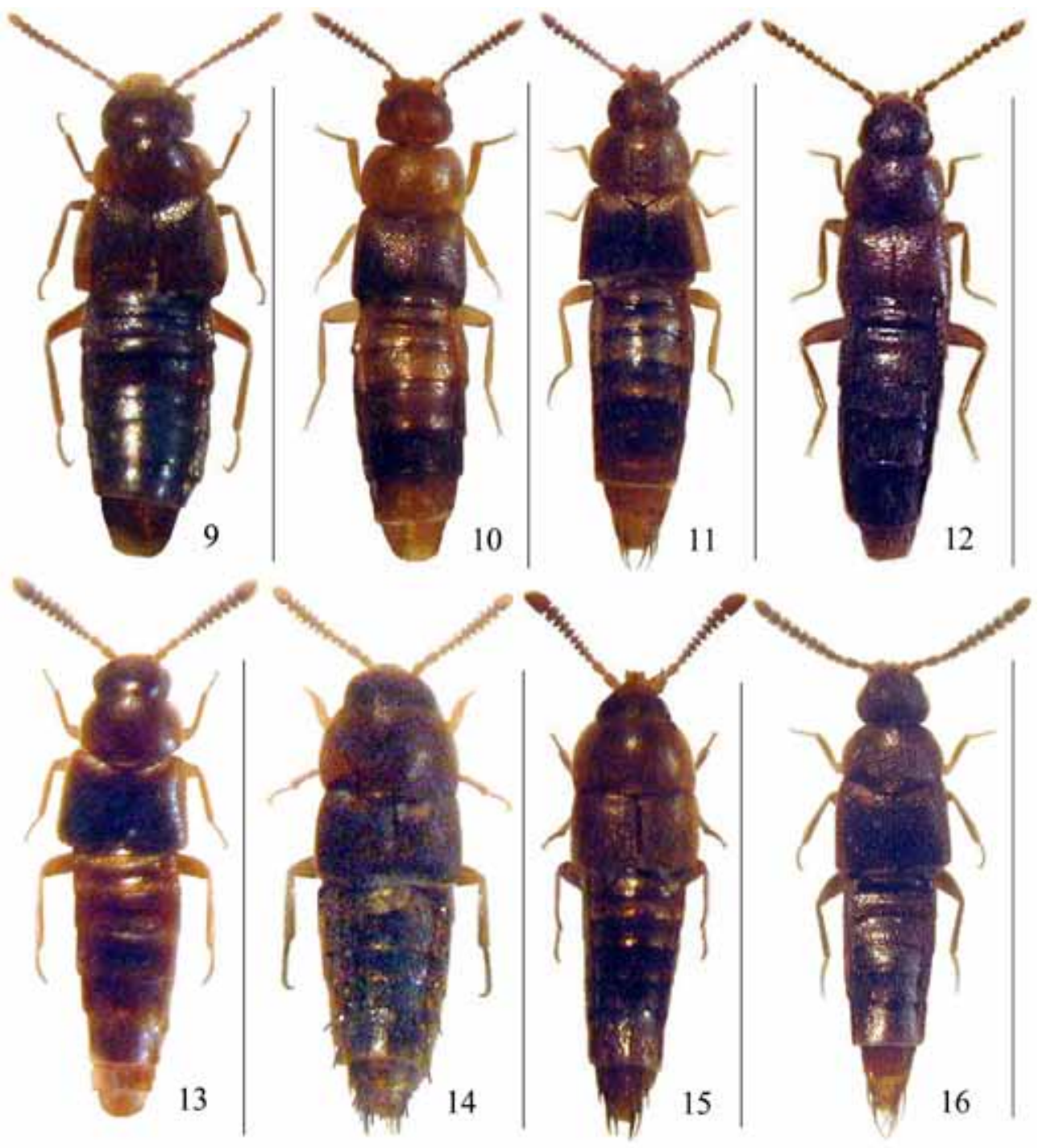

Figg. 9-16: Habitus. 9. Eutaenoglossa schuelkei $\mathrm{n}$. sp., scala $2 \mathrm{~mm}$; 10. Platorischna sinensis $\mathrm{n}$. sp., scala 1,6 mm; 11. Platorischna sichuanensis n. sp., scala $1,6 \mathrm{~mm} ; 12$. Lasiokoryphina sinica n. gen., n. sp., scala $1,7 \mathrm{~mm}$; 13 Medeterusa chinensis $\mathrm{n}$. sp., scala 2,1 mm; 14. Mimoxypoda sinica n.sp., scala 1,4 mm; 15. Mimoxypoda sinorum n. sp., scala 1,8 mm; 16. Litoglossa chinensis n. sp., scala 1,6 mm. 

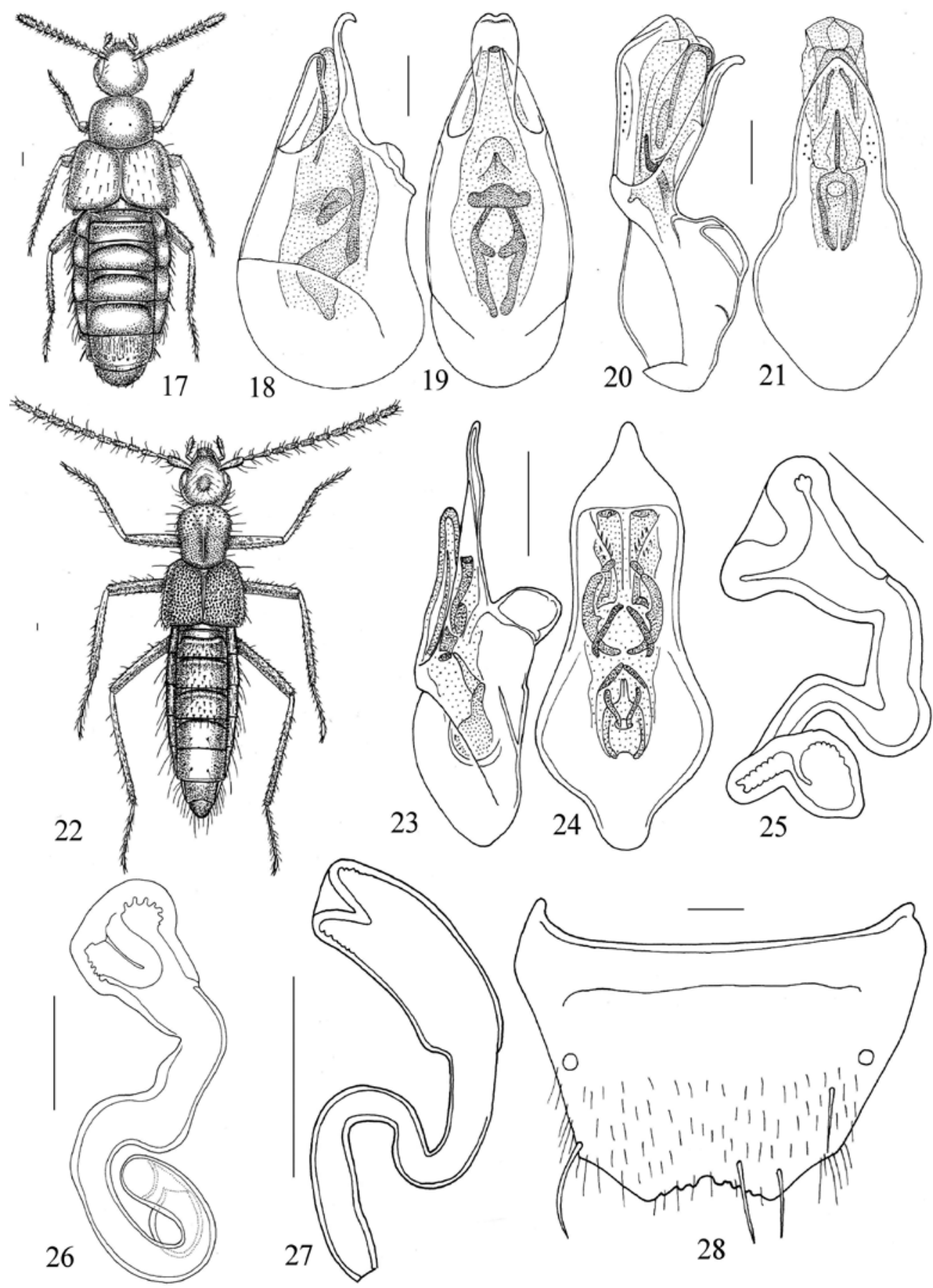

Figg. 17-28: Habitus, edeago in visione laterale e ventrale, spermateca e sesto urotergo libero della $\sigma^{\text {t. }}$. 17-19. Orphnebius uncinatus n. sp.; 20-21. Tetrabothrus chinensis n. sp.; 22-25. Drusilla damingensis n. sp.; 26. Drusilla cenwanglaomontis $\mathrm{n}$. sp.; 27-28. Drusilla gilva $\mathrm{n}$. sp., scale $0,1 \mathrm{~mm}$. 


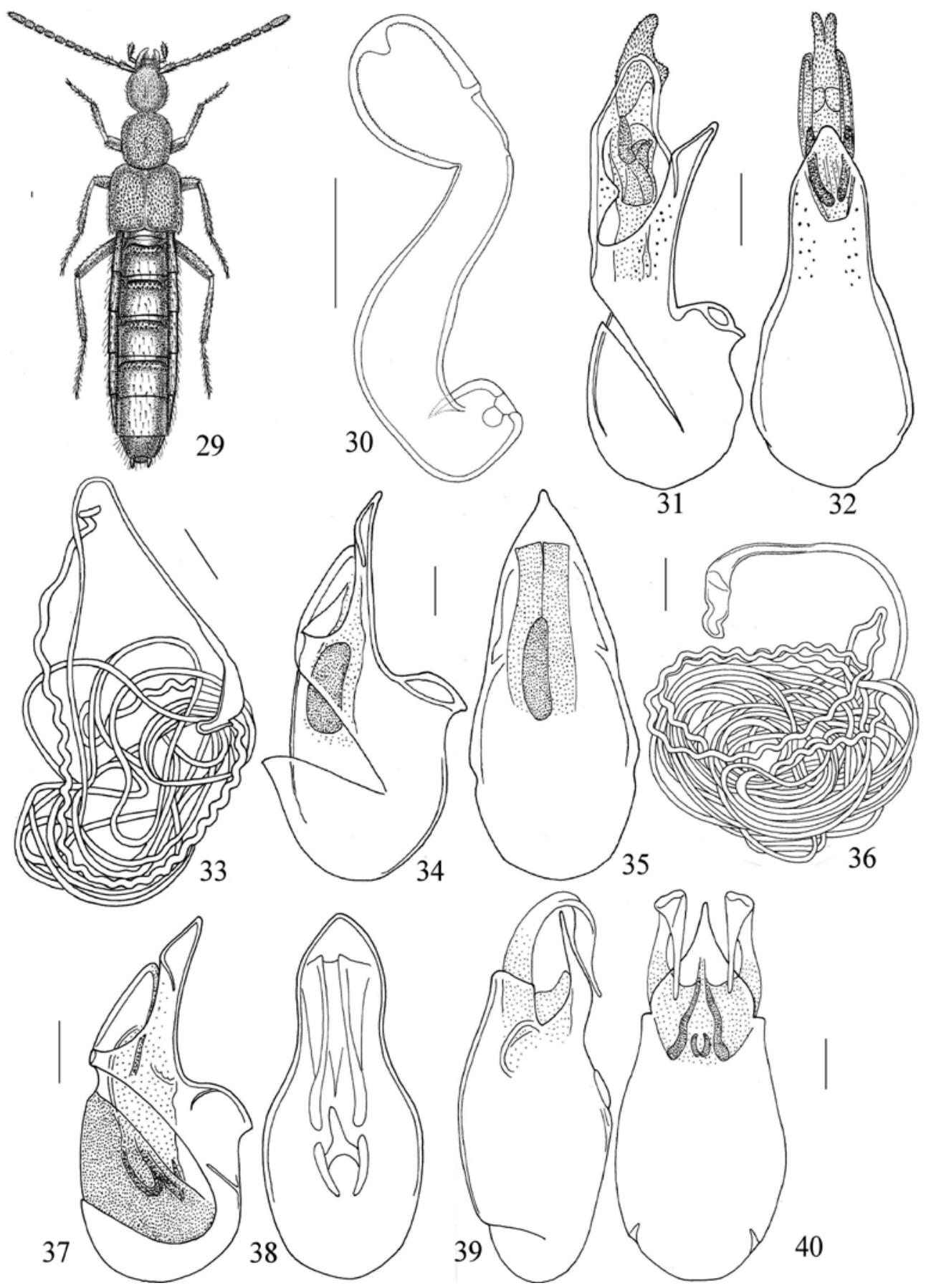

Figg. 29-40: Habitus, spermateca e edeago in visione laterale e ventrale. 29-30. Drusilla sinicola n. sp.; 31-33. Zyras (Zyras) qingchengensis n. sp.; 34-36. Zyras (Zyras) sichuanorum n. sp.; 37-38. Zyras (Zyras) yongshengensis $\mathrm{n}$. sp.; 39-40. Zyras (Rhynchodonia) diaoluomontis $\mathrm{n}$. sp., scale $0,1 \mathrm{~mm}$. 

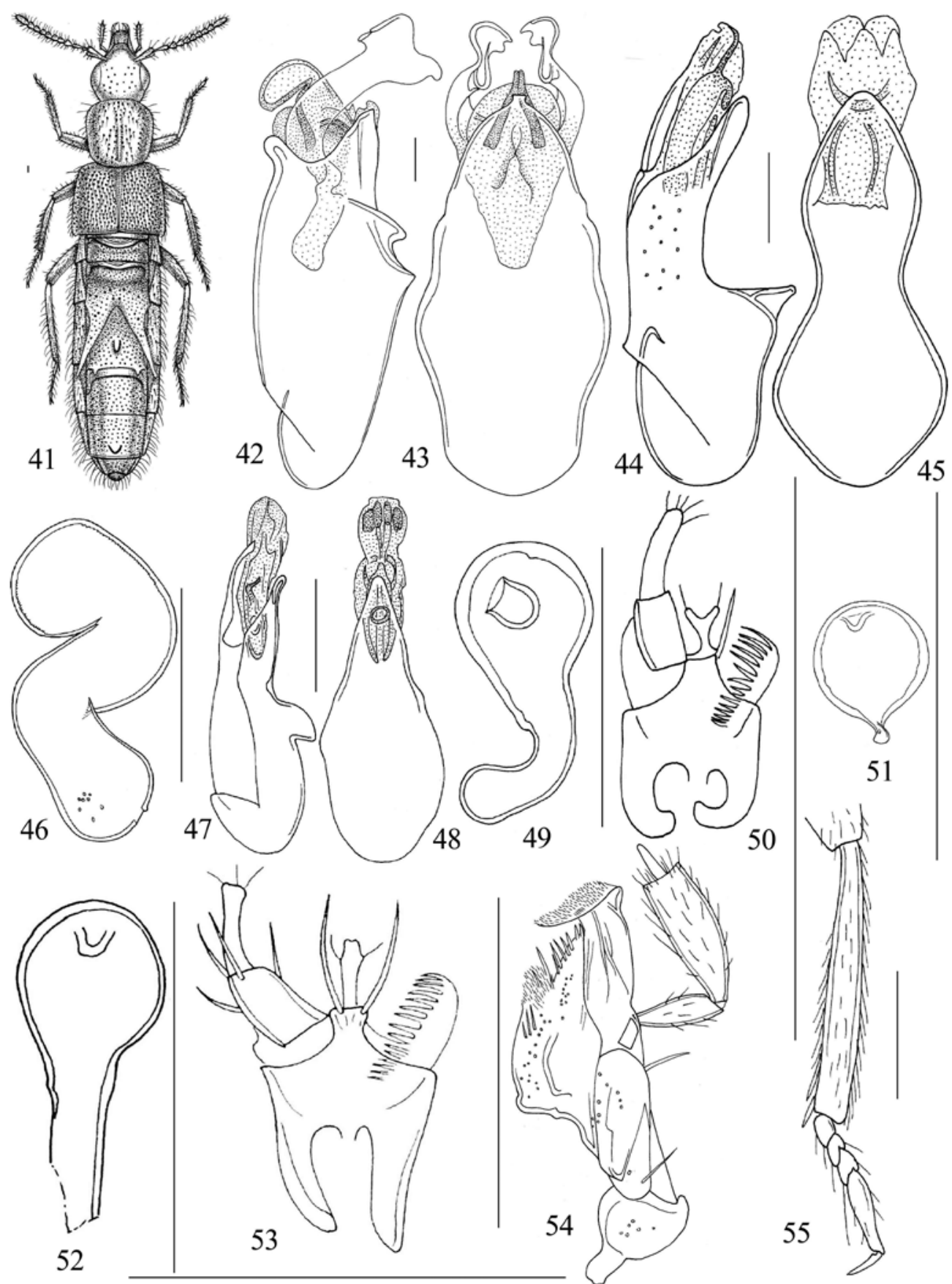

Figg. 41-55: Habitus, edeago in visione laterale e ventrale, spermateca, labio con palpo labiale, maxilla con palpo mascellare e protibia. 41-43. Zyras (Rhynchodonia) fellowesi n. sp.; 44-45. Pedinopleurus jiuwanmontis n. sp.; 46. Eutaenoglossa schuelkei n. sp.; 47-50. Platorischna sinensis n. sp.; 51. Platorischna sichuanensis n. sp.; 52-55. Lasiokoryphina sinica $\mathrm{n}$. gen., n. sp., scale $0,1 \mathrm{~mm}$. 


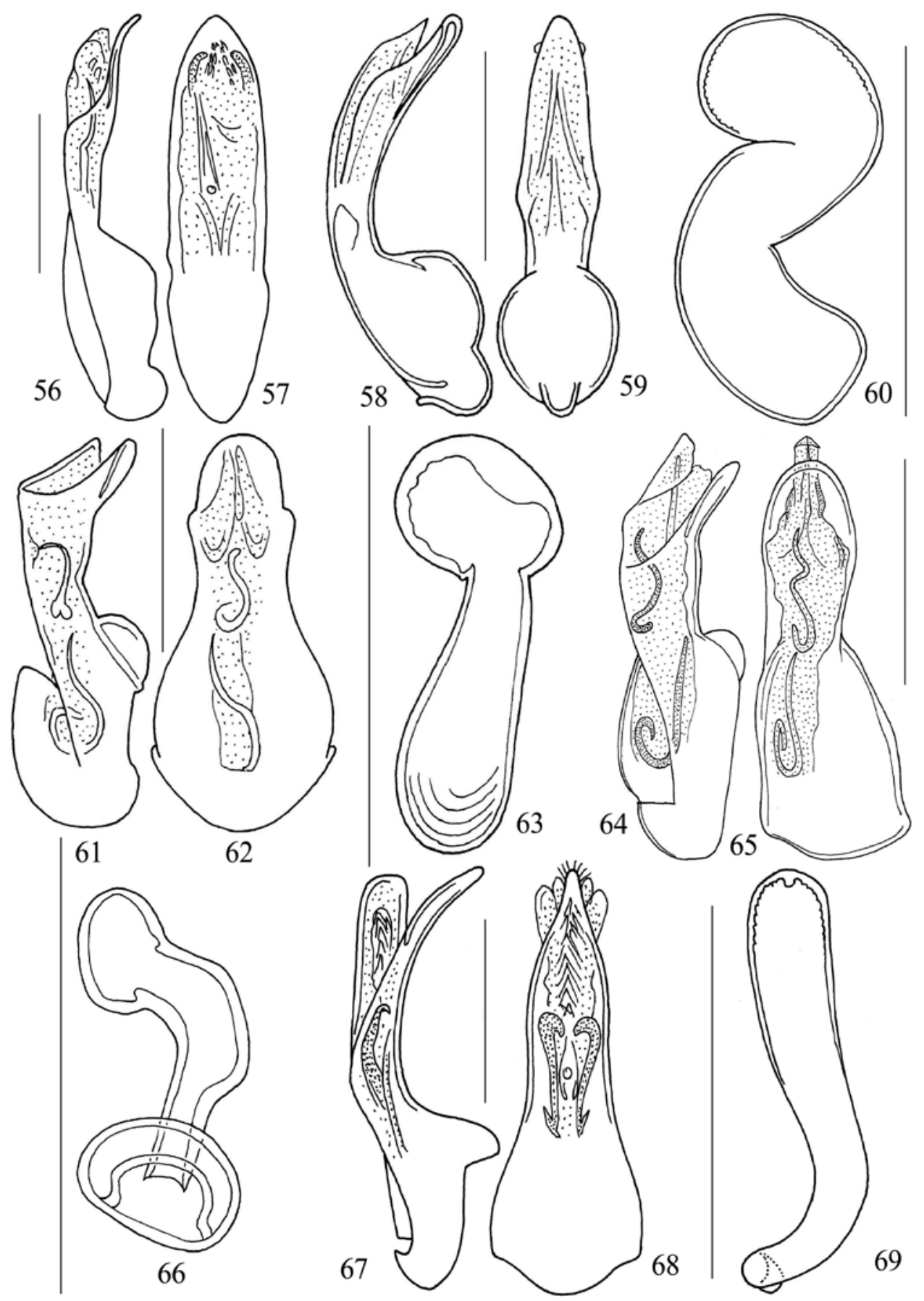

Figg. 56-69: Edeago in visione laterale e ventrale e spermateca. 56-57. Medeterusa chinensis n. sp.; 58-60. Mimacrotona chinensis n. sp.; 61-63. Mimoxypoda sinica n. sp.; 64-66. Mimoxypoda sinorum n. sp.; 67-69. Litoglossa chinensis $\mathrm{n}$. sp., scale 0,1 mm. 
Eppelsheim, E. 1895: Neue ostindische Staphylinen. 2. Stück. - Wiener Entomologische Zeitung 14: 53-70.

Fauvel, A. 1905: Staphylinidae exotiques nouveaux (3e partie). - Revue d'Entomologie 24: 113-147.

Fenyes, A. 1921: New Genera and Species of Aleocharinae with a polytomic Synopsis on the tribes. Bulletin of the Museum of Comparative Zoology 65: 17-36.

Fleming, J. 1821: Insecta: 41-46, pl. 85. - In: Supplement to the fourth, fifth and sixth editions of the Encyclopaedia Britannica, vol. 5. A. Constable, Edinburgh.

Leach, W. 1819: New Genera. - In: G. Samouelle, The Entomologist's useful Compendium: 1-496, London.

Maruyama, M. \& Kishimoto, T. 1999: A revision of the Japanese species of the genus Tetrabothrus (Coleoptera: Staphylinidae: Aleocharinae). - Elytra 27: 503-517.

Motschulsky, V. De. 1858: Énumération des nouvelles espèces de Coléoptères rapportées de ses voyages. - Bulletin de la Société impériale des Naturalistes de Moscou 3: 204-264.

PACE, R. 1984a: Aleocharinae della Thailandia e della Birmania riportate de C. de Rougemont. - Bollettino del Museo civico di Storia naturale di Verona 11: 427-468.

PACE, R. 1984b: Aleocharinae dell'Himalaya (Coleoptera: Staphylinidae) (LI Contributo alla conoscenza delle Aleocharinae). - Annales de la Société entomologique de France (N.S.) 20: 309-339.

PACE, R. 1987: Staphylinidae dell'Himalaya Nepalese. Aleocharinae raccolte dal Prof. Dr. Martens (Insecta: Coleoptera). - Courier Forschungs-Institut Senckenberg 93: 383-441.

PACE, R. 1991: Aleocharinae nepalesi del Museo di Ginevra. Parte V: Athetini (conclusione) e Thamiaraeini (Coleoptera, Staphylinidae) (111 ${ }^{\circ}$ Contributo alla conoscenza delle Aleocharinae). - Revue suisse de Zoologie 98: 803-863.

PACE, R. 1992: Aleocharinae nepalesi del Museo di Ginevra. Parte VI: Myrmedoniini (Coleoptera, Staphylinidae) ( $112^{\circ}$ Contributo alla conoscenza delle Aleocharinae). - Revue suisse de Zoologie 99: 125-145.

PaCE, R. 1993: Aleocharinae della Cina (Coleoptera Staphylinidae). - Bollettino del Museo civico di Storia naturale di Verona 17: 69-126.

PACE, R. 1998: Aleocharinae della Cina: Parte IV (Coleoptera, Staphylinidae). - Revue suisse de Zoologie 105: 911-982, 271 figg.

PACE, R. 2000: Aleocharinae della Thailandia (Coleoptera, Staphylinidae) $\left(144^{\circ}\right.$ Contributo alla conoscenza delle Aleocharinae). - Bollettino del Museo regionale di Scienze naturali di Torino 17: 39-86.

PAŚNIK, G. 2000: Three new species of Aleocharinae from Vietnam (Coleoptera, Staphylinidae). - Linzer Biologische Beiträge 32: 1057-1061, 8 figg.

Stephens, J. F. 1835: Illustrations of British Entomology. - Mandibulata 5: 369-448, London.

\section{Indirizzo dell'autore:}

Roberto Pace

Via Vittorio Veneto, 13

37032 Monteforte d'Alpone (Verona)

Italia

e-mail: pace.ent@tiscali.it

\section{Subject editor:}

Dr. L. ZerChE 